\title{
Nonanimal Stabilized Hyaluronic Acid for Tissue Augmentation of the Dorsal Hands: A Prospective Study on 38 Patients
}

\author{
Franck Marie P. Leclère $\cdot$ Esther Vögelin • \\ Serge Mordon · Justo Alcolea · Fernando Urdiales • \\ Frank Unglaub $\cdot$ Mario Trelles
}

Received: 6 January 2012/ Accepted: 17 July 2012/Published online: 6 October 2012

(C) Springer Science+Business Media, LLC and International Society of Aesthetic Plastic Surgery 2012

\begin{abstract}
Background Often ignored, hands are one of the most telltale signs of aging. This prospective study was initiated to evaluate the effect of subcutaneous hyaluronic acid (HA) injections in aging hands, with special attention to complications and long-term outcomes.

Methods Between January 2010 and December 2010, a total of 38 patients with skin phototypes II-IV and between 58 and 76 years old were treated with HA injection for aging hands. The quantity of injection never exceeded 1.0-1.5 ml HA per hand. A clinical follow-up was performed at 2 weeks, 4 weeks, 3 months, and 6 months after injection. Complications were reviewed for the whole series. At the first follow-up, 2 weeks after the procedure, ultrasound was carried out to determine if additional filling material was required. At each follow-up, patients were asked to fill out a satisfaction questionnaire.

Results Nine patients developed slight ecchymosis that disappeared after 1 week. No other complications were seen
\end{abstract}

F. M. P. Leclère $(\bowtie) \cdot$ E. Vögelin

Department of Plastic and Hand Surgery, Inselspital Bern, University of Bern, 3010 Bern, Switzerland

e-mail: franckleclere@yahoo.fr; franck.leclere@inserm.fr;

franck.leclere@insel.ch

F. M. P. Leclère $\cdot$ S. Mordon

INSERM, U 703 (French National Institute of Health and Medical Research), Université Lille Nord de France, Lille

University Hospital, CHRU, Lille, France

J. Alcolea $\cdot$ F. Urdiales $\cdot$ M. Trelles

Instituto Médico Vilafortuny, Cambrils, Spain

F. Unglaub

Department of Hand Surgery, Vulpiusklinik, Bad Rappenau, Germany in the series. Pain during the injection and discomfort after the procedure were minimal. At the 2-week follow-up, after ultrasound control, nine patients received a complementary injection. At each follow-up, overall patient satisfaction was high and was validated by clearance of rhytids, veins, bony prominences, and dermal and subcutaneous atrophy.

Conclusion Skin revitalization with injectable HA can improve the clinical appearance of the back of the hands. However, this therapy requires knowledge of the possible complications and their remediation as well as knowledge and respect of injected doses. Moreover, despite excellent results at each follow-up, the results of our series are not as good after 6 months, and a longer follow-up would be needed to determine if this procedure provides long-lasting benefit.

Level of Evidence III This journal requires that authors assign a level of evidence to each article. For a full description of these Evidence-Based Medicine ratings, please refer to the Table of Contents or the online Instructions to Authors www.springer.com/00266.

Keywords Hyaluronic acid · Aging hand · Hand rejuvenation - Tissue augmentation · Fillers

The face is frequently the focus of rejuvenation [1-5]. Often ignored, hands exhibit some of the most telltale signs of aging. Clinically, photoaging or extrinsic aging must be distinguished from intrinsic aging [6]. Intrinsically aging hands are characterized by rhytids, dermal and fat atrophy, visible color of tendons, prominent veins, and noticeable bony contours [7]. In the field of hand rejuvenation, therapies have focused mainly on reducing pigmentation through laser irradiation, intense pulsed light, chemical peels, and microabrasions [8]. Studies on corrections of the intrinsic aspects of aging hands are still limited and often 
involve autologous fat injections, sclerotherapy, phlebectomy, or skin excision $[9,10]$.

Hyaluronic acid (HA) is a linear polysaccharide comprising alternating glucuronic acid and $N$-acetylglucosamines residues and belongs to a group of substances known as glycosaminoglycans. Skin normally contains the highest concentration of hyaluronic acid within the body. However, with time, there is a progressive depletion of endogenous HA [11]. HA has been implicated in a number of processes during early wound healing, including cell migration and proliferation [12], organization of granulation tissue, stimulating angiogenesis, and moderating the inflammatory response $[12,13]$. After modification of HA into a more stabilized preparation, these properties have been used successfully by dermatologists and plastic surgeons in facial rejuvenation for restoring skin volume and improving its physiology. Its typical effects can last up to 9 months depending on the treated area. Despite the numerous studies evaluating this therapy on the face [1-5], there is still a paucity of studies $[14,15]$ describing its potential on hand rejuvenation. This prospective study was initiated to evaluate the effect of subcutaneous HA injections in aging hands, with special attention paid to complications, patient satisfaction, and outcomes, i.e., clearance of rhytids, veins, bony prominences, and dermal and subcutaneous atrophy.

\section{Materials and Methods}

\section{Patients}

This clinical study protocol was reviewed and approved by the Ethics Committee of our institution. Thirty-eight patients with skin phototypes II-IV and between 58 and 76 years old (average $=69 \pm 4$ years) were enrolled. They signed an informed consent form for the study and injection after having been informed of all pertinent details.

\section{Filler}

A HA-based filler of nonanimal origin (Cristal 2, DP Italia, Italy, and Irradia, Spain) was injected for hand rejuvenation. The molecular size of this HA is $300 \mu \mathrm{m}$ and it has a viscosity of $20,000 \mathrm{mPa}$. The filler is composed of $24 \mathrm{mg} / \mathrm{ml}$ sodium hyaluronate cross-linked hyaline gel type. One important characteristic of this HA is that although it has large molecules, it also has thixotropic properties that enable its injection with a $27 \mathrm{G}$ cannula. This is not possible with standard high-viscosity HAs because high viscosity directly impacts the resistance of the fluid and its ability to pass through a small-diameter cannula. In the case of the particular product used in this study, viscosity was changed by shear stress or pressure applied tangentially to the HA contained in the syringe. The HA gel product becomes thinner when stressed by the pressure placed on the syringe at the time of the injection, causing it to pass with ease through the needle for precise deposition into the tissue. Due to the thixotropic property of the HA, it takes a fixed amount of time to transform into a firmer, more solid viscous equilibrium once it is introduced into the skin and reaches dermal layers. During this time, pressure is applied using a maneuver in which the injection molds the product in the area, easily obtaining the desired shape. There is usually no need to inject more than $1.0-1.5 \mathrm{ml}$ of HA per hand. In cases of significant skin laxity and fat atrophy, another injection is given after 2 weeks to achieve optimal filling. This allows for accurate control of the amount of HA needed and avoids the typical aspect of dorsum edema after treatment.

\section{Injection Technique}

Once anesthesia ( $2 \%$ lidocaine) is injected at the point where the cannula is to be introduced, a $21 \mathrm{G}$ needle is used to pierce the skin in the fold that appears when the hand is hyperextended. This cannula entry point permits easy access to the spaces between the tendons (Fig. 1). If the depression is more distal, then needle access is between the second and third and between the third and fourth fingers. In these instances, a $27 \mathrm{G}$ blunt and flexible cannula (40 mm long), with a lateral opening to allow the filler out, is introduced. To avoid damaging the synovial layers of the tendon, the skin is pinched with the thumb and index fingers. This is easy to do because of the laxity presented by aged hands. Once the cannula is inserted between the fingers and the correct area is selected, it is moved in a gentle longitudinal swinglike motion, avoiding lateral side-to-side movement so as not to damage the superficial nerves. The flexibility of the cannula causes it to bend easily and therefore help in the maneuver. Also, its length makes it possible to reach the various distances of the hand dorsum.

\section{Postoperative Care}

Patients were advised to avoid strenuous hand activity and not let the hands hang down for too long as the effects of gravity would cause painful and uncomfortable edema on the back of the hands. Patients received $15 \mathrm{mg}$ of prednisolone immediately after treatment and $24 \mathrm{~h}$ later.

\section{Objective Assessment}

At the 2-week, 4-week, 3-month, and 6-month follow-up, clinical examination was performed and complications were carefully recorded, with special attention given to 
Fig. 1 a Control of the cannula in the space noticed between the tendons. b HA injection using gentle longitudinal swing-like movements

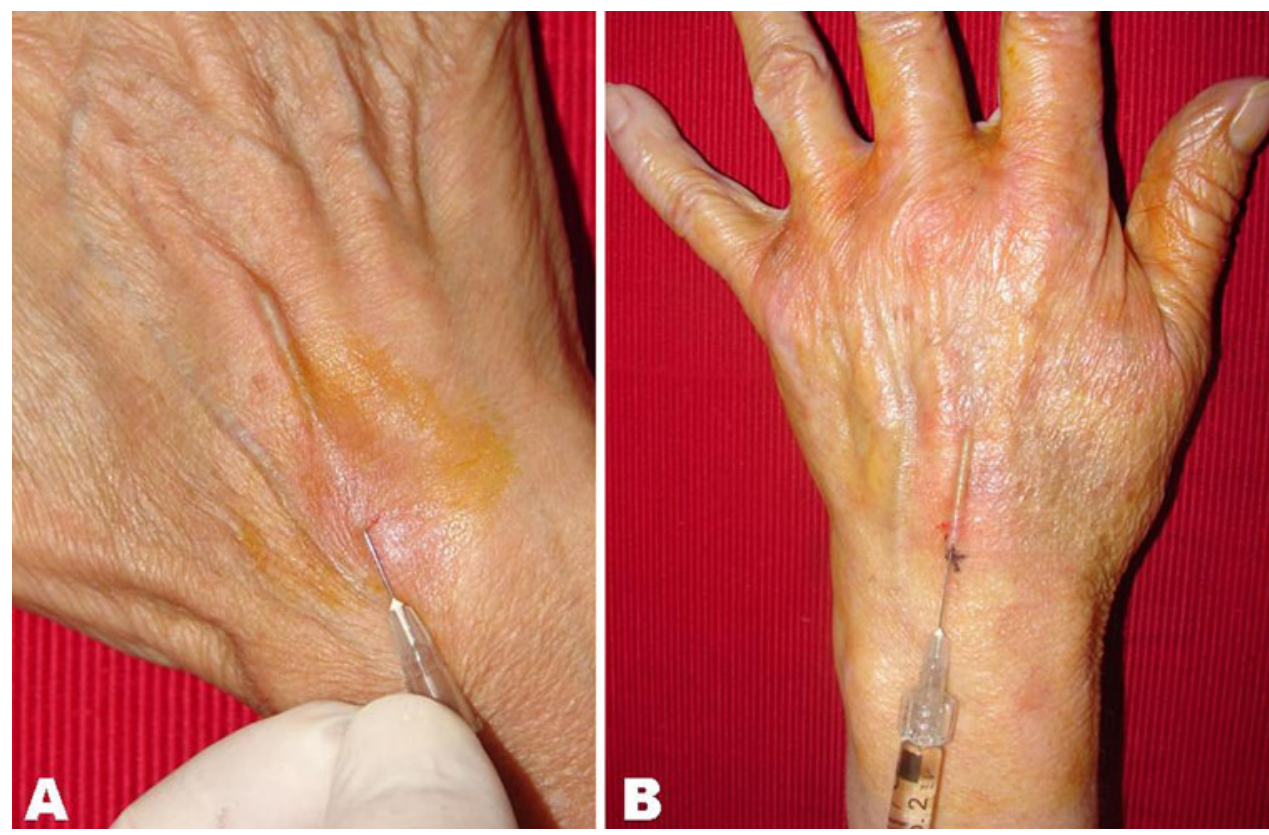

the questionnaire shown in Table 1. The answers were given a numerical score, where 0 , no pain/tingling/bruising/discomfort; 1, slight pain/tingling/bruising/discomfort; 2, moderate pain/tingling/bruising/discomfort; 3, severe pain/tingling/bruising/discomfort; and 4, very severe pain/ tingling/bruising/discomfort. Inability to work was carefully recorded $(0=$ nil, $1=$ one day, $2=$ two days, $3=$ three days, $4=$ more than 3 days). Patients were asked to score their improvement at each follow-up period (from 0 to $100 \%$ ) on a visual scale [Expectations met: 4 $($ excellent $)=90-100 \% ; 3($ good $)=70-89 \% ; 2$ (regular) $=40-69 \% ; 1$ (poor) $=0-39 \%$. At the 6 month follow-up, patients were asked whether they would recommend the procedures to others.

\section{Statistical Analysis}

The prospective study included 38 patients with aging hands compared prior to and 2 weeks, 4 weeks, 3 months, and 6 months after HA injection. The different measurements are reported in Tables 2, 3, 4. An ANOVA with main variables of time and the individual patient was performed to show differences between the four postinjection points (patient effects were isolated). The following parameters were scored: clearance of rhytids, veins, bony prominences, and dermal and subcutaneous atrophy. A $p<0.05$ was considered to be statistically significant. Student's $t$ test (two dependent samples) was subsequently used to show differences between the measurements 2 weeks after HA injection and each of the latter time points for each parameter. Statistical analysis was performed using the SPSS Stat program (SPSS, Inc., Chicago, IL).

Patients were asked to rate their level of discomfort while the hands were anesthetized and after the procedure using 
Table 1 Questionnaire used for our study

Table 2 Complaints during the injection, discomfort after the procedure, and interference with daily activities in our series of 38 patients

Table 3 Subjective improvement in our series of 38 patients

Did you experience pain, tingling, or bruising during the injection?

$\square$ NO

$\square$ YES:

Pain: $\square$ Slight $\square$ Moderate $\square$ Severe $\square$ Very severe

Tingling: $\square$ Slight $\square$ Moderate $\square$ Severe $\square$ Very severe

Bruising: $\square$ Slight $\square$ Moderate $\square$ Severe $\square$ Very severe

Did you feel discomfort immediately after the procedure and during the first two weeks?

$\square$ NO

$\square$ Slight $\square$ Moderate $\square$ Severe $\square$ Very severe

Has the procedure interfered with your daily activities?

$\square$ NO

$\square$ YES:

$\square$ Slight $\square$ Moderate $\square$ Severe $\square$ Very severe

Has the procedure prevented you from working?

$\square$ NO

$\square$ YES:

$\square 1$ day $\square 2$ days $\square 3$ days $\square>3$ days

How do you perceive the improvement brought by the procedure?

(please mark it on the visual scale)

2 weeks $0 \% \rightarrow 100 \%$ (excellent)

1 month $0 \% \rightarrow 100 \%$ (excellent)

3 months $0 \% \rightarrow 100 \%$ (excellent)

6 months $0 \% \rightarrow 100 \%$ (excellent)

Would you recommend this treatment to others?

$\square$ YES

$\square$ NO

\begin{tabular}{lrrrlrc}
\hline & \multicolumn{3}{l}{ During injection } & & During the first 2 weeks & Interference with daily activities \\
\cline { 2 - 4 } & Pain & Tingling & Bruising & Discomfort & 36 \\
\hline 0: No & 35 & 37 & 34 & 36 & 2 \\
1: Slight & 2 & 1 & 4 & 2 & 0 \\
2: Moderate & 1 & 0 & 0 & 0 & 0 \\
3: Severe & 0 & 0 & 0 & 0 & 0 \\
4: Very severe & 0 & 0 & 0 & 0 & 0 \\
\hline
\end{tabular}

\begin{tabular}{lllllll}
\hline Efficacy & $\%$ & Score & 2 weeks & 1 month & 3 months & 6 months \\
\hline Excellent & $90-100$ & 4 & 33 & 32 & 31 & 26 \\
Good & $70-89$ & 3 & 3 & 4 & 4 & 7 \\
Regular & $40-69$ & 2 & 2 & 2 & 3 & 5 \\
Poor & $10-39$ & 1 & 0 & 0 & 0 & 0 \\
Mean \pm SD $(p)$ & & & $3.8 \pm 0.5$ & $3.8 \pm 0.5(0.16)$ & $3.7 \pm 0.6(0.08)$ & $3.5 \pm 0.7(<0.01)$ \\
\hline
\end{tabular}


Table 4 Effect of the therapy on rhytids, veins, bony prominences, and dermal and subcutaneous atrophy

\begin{tabular}{|c|c|c|c|c|c|c|c|}
\hline & Efficacy & $\%$ & Score & 2 weeks & 1 month & 3 months & 6 months \\
\hline \multirow[t]{5}{*}{ Clearance of rhytids } & Excellent & $90-100$ & 4 & 29 & 32 & 27 & 23 \\
\hline & Good & $70-89$ & 3 & 5 & 3 & 7 & 9 \\
\hline & Regular & $40-69$ & 2 & 4 & 3 & 4 & 6 \\
\hline & Poor & $10-39$ & 1 & 0 & 0 & 0 & 0 \\
\hline & Mean \pm SD $(p)$ & & & $3.7 \pm 0.6$ & $3.7 \pm 0.5(0.02)$ & $3.6 \pm 0.7(0.006)$ & $3.4 \pm 0.8(0.006)$ \\
\hline \multirow[t]{5}{*}{ Clearance of veins } & Excellent & $90-100$ & 4 & 31 & 34 & 28 & 24 \\
\hline & Good & $70-89$ & 3 & 4 & 3 & 5 & 9 \\
\hline & Regular & $40-69$ & 2 & 3 & 1 & 5 & 5 \\
\hline & Poor & $10-39$ & 1 & 0 & 0 & 0 & \\
\hline & Mean \pm SD $(p)$ & & & $3.7 \pm 0.6$ & $3.9 \pm 0.4(0.01)$ & $3.6 \pm 0.7(0.001)$ & $3.5 \pm 0.7(0.02)$ \\
\hline \multirow[t]{5}{*}{ Clearance of bony prominences } & Excellent & $90-100$ & 4 & 31 & 30 & 29 & 26 \\
\hline & Good & $70-89$ & 3 & 4 & 6 & 6 & 8 \\
\hline & Regular & $40-69$ & 2 & 3 & 2 & 3 & 3 \\
\hline & Poor & $10-39$ & 1 & 0 & 0 & 0 & 1 \\
\hline & Mean \pm SD $(p)$ & & & $3.7 \pm 0.6$ & $3.7 \pm 0.6(0.5)$ & $3.7 \pm 0.6(0.08)$ & $3.6 \pm 0.7(0.02)$ \\
\hline \multirow[t]{5}{*}{ Dermal and subcutaneous atrophy } & Excellent & $90-100$ & 4 & 27 & 31 & 26 & 22 \\
\hline & Good & $70-89$ & 3 & 7 & 4 & 5 & 9 \\
\hline & Regular & $40-69$ & 2 & 4 & 3 & 6 & 5 \\
\hline & Poor & $10-39$ & 1 & 0 & 0 & 1 & 2 \\
\hline & Mean \pm SD $(p)$ & & & $3.6 \pm 0.7$ & $3.7 \pm 0.6(0.01)$ & $3.5 \pm 0.9(<0.001)$ & $3.3 \pm 0.9(0.01)$ \\
\hline
\end{tabular}

\section{Results}

\section{Complications}

Except mild ecchymosis in 9 patients that resolved within 1 week, there were no complications in our series (Figs. 2, $3,4,5)$.

\section{Subjective Assessment}

The incidence of pain, tingling, and bruising during the procedure is reported in Table 2. Slight discomfort post procedure was reported by two patients. Return to normal activities never took longer than 1 day and mean downtime was 0.1 day. Mean subjective improvement at 2 weeks, 1 month, 3 months, and 6 months (Table 3) was $3.8 \pm 0.5$, $3.8 \pm 0.5(p=0.16), 3.7 \pm 0.6(p=0.08)$, and $3.5 \pm 0.7$ $(p<0.01)$, respectively. Of the 38 patients, 29 would recommend this treatment. The remaining 9 patients stated they would not recommend this procedure because they experienced unexpected ecchymosis (Figs. 6, 7).

\section{Ultrasound Assessment}

At the 2 week follow-up, ultrasound revealed insufficient filling in the fold of the second intermetacarpal space for
Fig. 2 59-year-old patient before (a) and 6 months after HA injection (b)
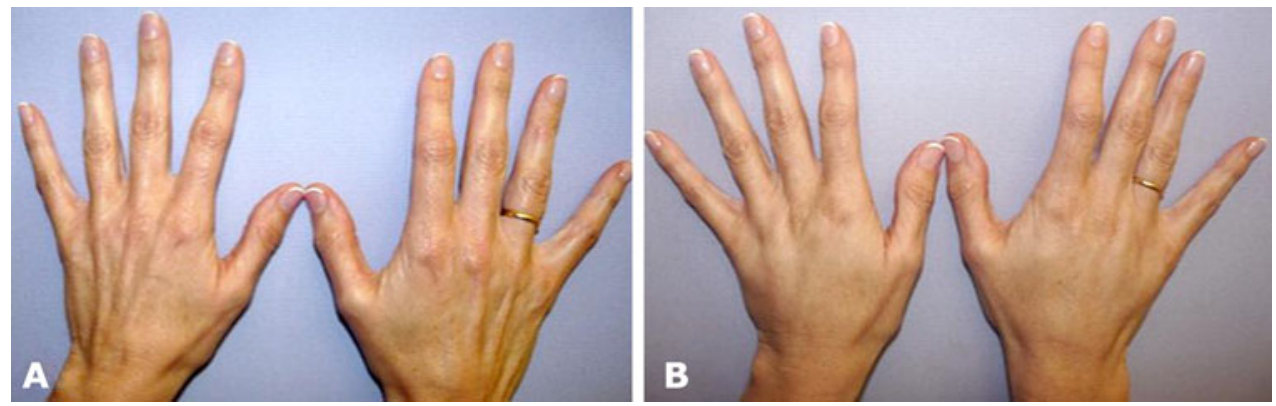
Fig. 3 66-year-old patient before (a) and 6 months after HA injection (b)
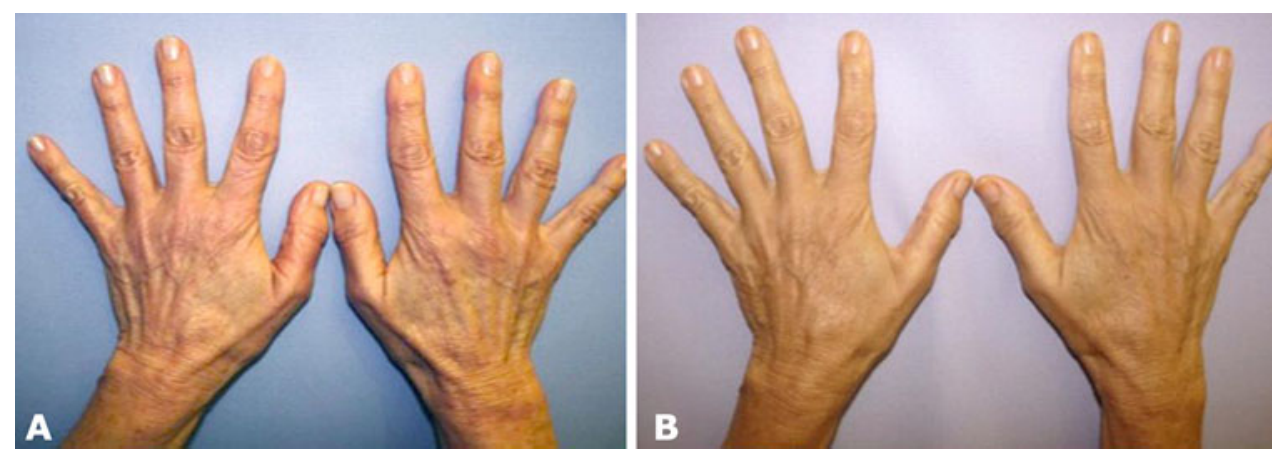

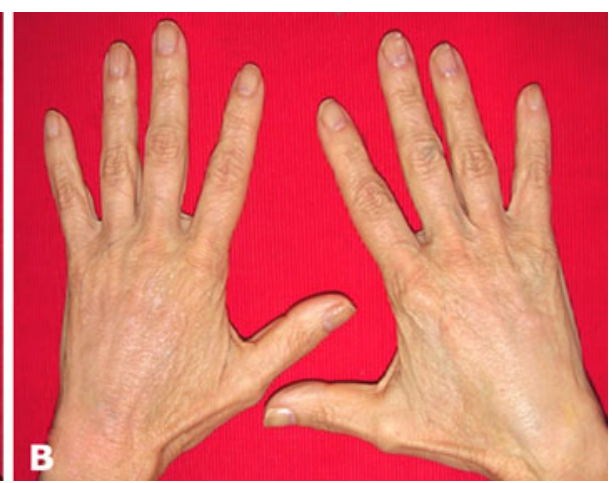

Fig. 4 58-year-old patient before (a) and 6 months after HA injection (b)

Fig. 5 76-year-old patient before (a) and 6 months after HA injection (b)
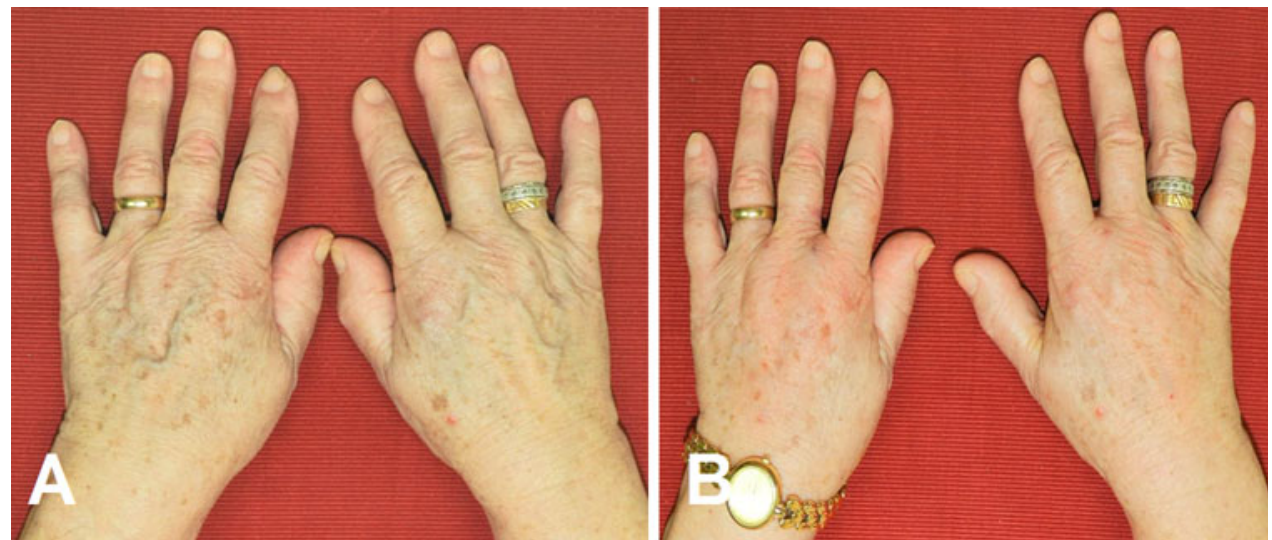

one patient, in the fold of the second and third intermetacarpal spaces for seven patients, and in the fold of the fourth intermetacarpal space for one patient. These nine patients received a complementary injection because of insufficient results after the first treatment. For the nine patients who were reinjected, the follow-up began at the time of the second injection.

\section{Objective Assessment}

The mean scores at 2 weeks, 1 month, 3 months, and 6 months (Table 4) were $3.7 \pm 0.6,3.7 \pm 0.5,3.6 \pm 0.7$, and $3.4 \pm 0.8$, respectively, for clearance of rhytids. The ANOVA shows significant differences $(p<0.001)$ in scores between the different points in time for this 


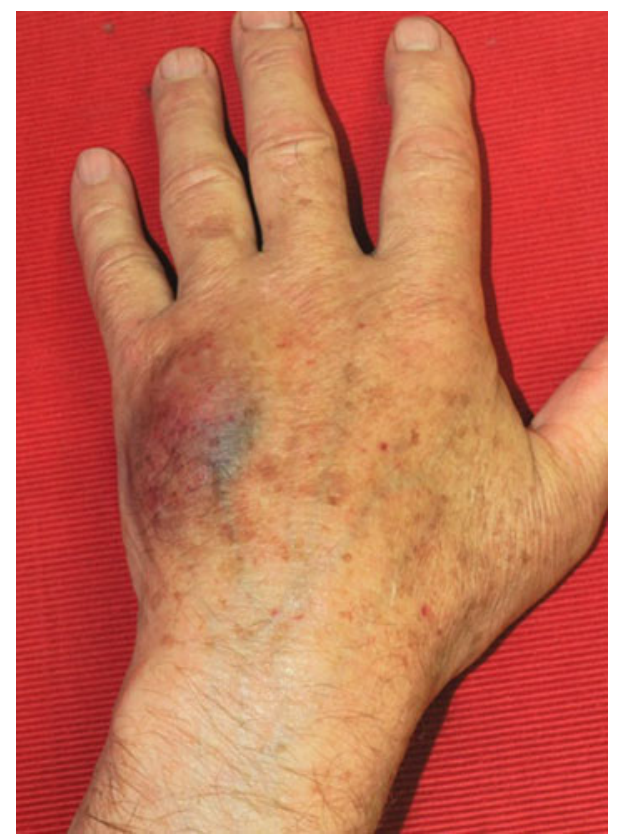

Fig. 6 Mild ecchymosis occurring after HA injection at the level of the fourth intermetacarpal space

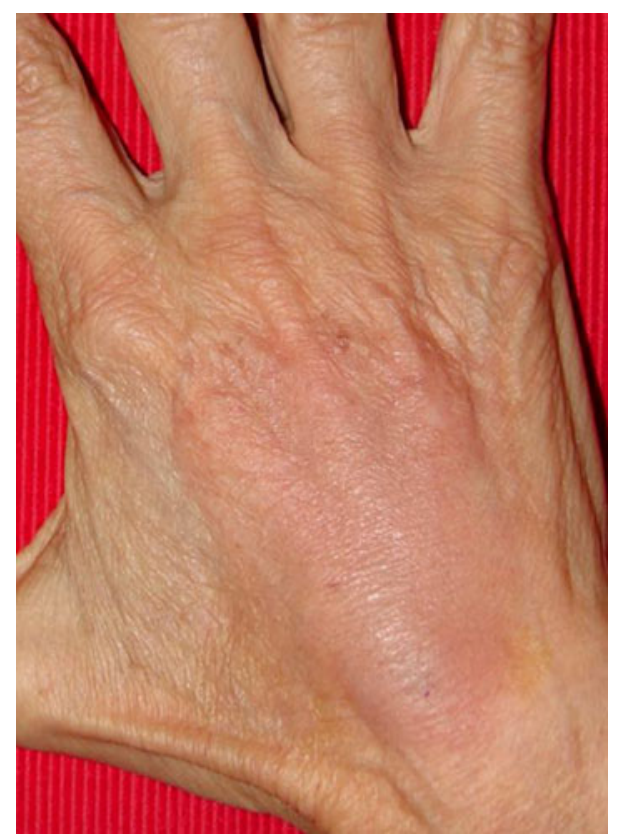

Fig. 7 Mild ecchymosis occurring after HA injection at the level of the second and third intermetacarpal spaces

parameter. The $p$ values for the paired $t$ tests were 2 weeks versus 1 month: $0.044,2$ weeks versus 3 months: 0.160 , and 2 weeks versus 6 months: 0.003 .

Mean subjective scores at 2 weeks, 1 months, 3 months, and 6 months (Table 4) were $3.7 \pm 0.6,3.9 \pm 0.4$, $3.6 \pm 0.7$, and $3.5 \pm 0.7$, respectively, for clearance of veins. The ANOVA shows significant differences $(p<0.001)$ in scores between the different points in time for clearance of veins. The $p$ values for the paired $t$ tests were 2 weeks versus 1 month: $0.023,2$ weeks versus 3 months: 0.023 , and 2 weeks versus 6 months: 0.002 .

Mean subjective scores at 2 weeks, 1 months, 3 months, and 6 months (Table 4) were $3.7 \pm 0.6,3.7 \pm 0.6$, $3.7 \pm 0.6$, and $3.6 \pm 0.7$, respectively, for clearance of bony prominences. The ANOVA analysis shows significant differences $(p=0.001)$ in scores between the different points in time for this parameter. The $p$ values for the paired $t$ tests were 2 weeks versus 1 month: 1.000, 2 weeks versus 3 months: 0.160 , and 2 weeks versus 6 months: 0.006 .

Mean subjective scores at 2 weeks, 1 months, 3 months, and 6 months (Table 4) were $3.6 \pm 0.7,3.7 \pm 0.6$, $3.5 \pm 0.9$, and $3.3 \pm 0.9$, respectively, for clearance of dermal and subcutaneous atrophy. The ANOVA shows significant differences $(p<0.001)$ in scores between the different points in time for this parameter. The $p$ values for the paired $t$ tests were 2 weeks versus 1 month: 0.023 , 2 weeks versus 3 months: 0.023 , and 2 weeks versus 6 months: 0.001 .

\section{Discussion}

Often neglected by plastic surgeons, hands exhibit the most telltale signs of aging. Resurgence in the treatment of aging hands, in both the aesthetics of the hand and volumetric options for intrinsic aging, is occurring due to (1) patient demand; (2) the current literature on face rejuvenation [1-5], and (3) the creation of dedicated societies like the Multidisciplinary European Group for Aesthetics of the Hand (MEGAHAND). However, most of the therapies have concentrated on keratosis, solar lentigo, solar purpura, and rhytids. These therapies include sclerotherapy, intense pulsed light, laser, and microdermabrasion [8, 14]. Unfortunately, the results are unpredictable and only temporary. Hypertrophic scarring, hyperpigmentation, and dyschromia are known complications of these therapies, especially in darker-skinned patients [6].

Because aging hands are more complex and include not only rhytids and skin appearance but also dermal and fat atrophy and veins and bony prominences, a different approach is required. Studies on the restoration of a more youthful fullness of the hands that focus on all of these characteristics are still limited and often involve skin excision, sclerotherapy, phlebectomy, and autologous fat injections. Excision and redraping of lax skin recreates a tight, youthful skin envelope, increasing the visibility of the underlying structures and thus exaggerating the initial problem of soft tissue loss [6, 16]. Sclerotherapy and phlebectomy may temporarily reduce visible veins, but due 
to impairment of venous return, edema formation and venous stasis may affect the patient permanently [6]. Because the hand lacks efficient deep venous drainage, the remaining veins will likely engorge over time. Fat injections to augment low-volume areas and soften the outline of deeper structures seem to be very promising $[6,9,10$, $16,17]$; however, since this is invasive therapy, it has led to some teams testing other fillers such as collagen or hyaluronic acid [15].

Hyaluronic acid is a glycosaminoglycan found in epithelial and connective tissue. HA has been implicated in a number of processes during early wound healing, including cell migration and proliferation [12], organization of granulation tissue, stimulation of angiogenesis, and moderation of the inflammatory response [13]. In the field of hand rejuvenation, the effectiveness of $\mathrm{HA}$ is based on its biocompatibility and its viscoelastic properties. However, due to its half-life of 1-2 days before enzymatic degradation, a chemical reticulation is necessary to obtain a waterinsoluble viscoelastic polymer with improved resistance to enzymatic reaction [14]. Our results after injection of nonanimal stabilized HA in the dorsum of the hands have shown significant clearance of rhytids, veins, bony prominences, and dermal and subcutaneous atrophy for all periods of observation. These results are partly explained by the fact that HA is able to hold up to 500-1,000 times its own weight of water in the extracellular matrix of the dermis [18]. In this context, Williams et al. [14], using a suction elasticity meter and moisture-measuring device, have clearly demonstrated an increase in cutaneous elasticity and stratum corneum hydration after stabilized HA injection in the dorsum of the hand. Their studies confirmed the previous report from Kerscher et al. [19] who used nonanimal stabilized HA for revitalization of the face, with excellent results for volume restoration, skin elasticity, and reduction in skin surface roughness. Moreover, Yoneda et al. [20], in an animal model, highlighted that exogenous stabilized HA acts as a modulator of dermal fibroblast proliferation that modifies indirectly the synthesis of collagen. Finally, Wang et al. [21] demonstrated that nonanimal stabilized HA injected into the dermal matrix is able to enhance the stimulation of de novo synthesis of type I collagen, possibly mediated by mechanical stretching and consequent activation of collagen-producing fibroblasts in the dermis.

Our results on the clearance of rhytids, veins, bony prominences, and dermal and subcutaneous atrophy are corroborated by the extremely high satisfaction of patients for each period of observation: they considered the results good to excellent in about $87 \%$ (6 months) to $92 \%$ (3 months) of cases, depending on the follow-up period. Moreover, $71 \%$ of them would recommend this therapy to others. The remaining nine patients $(29 \%)$ declared that they would not recommend this therapy because they experienced ecchymosis for which they were not prepared. This shows the consequences of even minor complications in highly demanding patients. Finally, complaints experienced during the injection phase are minimal in this study. Such subjective data, particularly the analysis of pain during injection, until now have not been reported in the literature [14].

In our study, in addition to the excellent results and the high satisfaction of the patients, there are very few complications. Complications included five instances of ecchymosis, which resolved after 10 days, and no patient's ability to work was hampered as the average down time was less than 1 day. Even though there were only a few complications in our study, they must be kept in mind by all operators [22]. There are indeed two types of exogenous stabilized HA: that of animal origin obtained by extraction and the nonanimal type that we used, obtained by fermentation. The first type carries a risk of allergic reaction related to the protein content, and the second can cause immunological reactions related to the presence of endotoxin [23]. In our experience, knowledge of the product is required but it is by no means enough in order to perform these injections for the following reasons: the studies of Lowe et al. [23], Brody et al. [24], and Lupton et al. [25] showed the risk of hypersensitivity reaction related to the use of these products. They must be handled by plastic surgeons or dermatologists who understand the potential complications and know how to manage them [26]. The use of hyaluronidase, which is the first tool that acts at the site of local injections to break down and hydrolyze hyaluronic acid, should be familiar to the operator. Moreover, knowledge and respect of doses is fundamental: In our experience, there is usually no need to inject more than 1.0-1.5 ml of HA per hand. In cases of significant skin laxity and fat atrophy, we repeated the injection after 2 weeks to achieve optimal filling. This allows for accurate control of the amount of HA needed and avoids the typical aspect of dorsum edema and ecchymosis after treatment. Finally, frequent follow-ups are necessary to assess the effectiveness of treatment and the possible occurrence of complications. In our study, a statistically significant difference was found between the 3 month and the 6 month follow-up regarding subjective improvement. This monitoring can also be used to repeat the injection if necessary. Williams et al. [14] confirmed this by showing that the injection should be repeated after 4 months. This frequency rate is much higher than that found in the literature for injections in the face, probably because of the different physiology of the skin at the two locations, but also because the hands are washed more frequently.

Although our study shows an improvement in aging hands by injections of stabilized HA, it also presents 
methodological limitations. First, a control group is clearly lacking. Because of our wealth of experience with the nonanimal stabilized HA product and the lack of complications, we did not feel inclined to use another product. Second, the follow-ups at 3 and 6 months appear too far apart and more frequent follow-ups would have been appropriate to determine precisely (as in the study of Williams et al. [14]) the best time for reinjection. In this context it is important to emphasize that the results at 6 months are not as good as those at 2 weeks after the procedure, and a longer follow-up is needed to determine if this procedure provides long-lasting benefit. Moreover, this study focused on the restoration of volume and largely omits the changes in skin physiology [15]. We preferred to focus our efforts on the subjective evaluation of the injection that has been largely neglected in the literature. Finally, given the necessary repetition of this type of treatment to maintain the rejuvenation, it would have been interesting to evaluate the risk of complications after regular injections. A multicenter study is underway on a large cohort of patients.

\section{Conclusion}

Skin revitalization with injectable HA can improve the clinical appearance of the back of the hand. However, this therapy necessitates knowledge of the possible complications and their treatments and knowledge and respect of injected doses. Moreover, despite excellent results at each follow-up, the results of our series are not as good after 6 months, and a longer follow-up would be needed to determine if this procedure is of long-lasting benefit.

Conflict of interest The authors have no conflicts of interest to disclose.

\section{References}

1. Wu W, Carlisle I, Huang P, Ribé N, Russo R, Schaar C, Verpaele A, Strand A (2010) Novel administration technique for large-particle stabilized hyaluronic acid-based gel of nonanimal origin in facial tissue augmentation. Aesthetic Plast Surg 34:88-95

2. Lemperle G, Sadick NS, Knapp TR, Lemperle SM (2010) ArteFill permanent injectable for soft tissue augmentation: II. Indications and applications. Aesthetic Plast Surg 34:273-286

3. Kane MA (2005) Treatment of tear trough deformity and lower lid bowing with injectable hyaluronic acid. Aesthetic Plast Surg 29:363-367

4. Bergeret-Galley C, Latouche X, Illouz YG (2001) The value of a new filler material in corrective and cosmetic surgery: DermaLive and DermaDeep. Aesthetic Plast Surg 25:249-255
5. Olenius M (1998) The first clinical study using a new biodegradable implant for the treatment of lips, wrinkles, and folds. Aesthetic Plast Surg 22:97-101

6. Jakubietz RG, Jakubietz MG, Kloss D, Gruenert JG (2005) Defining the basic aesthetics of the hand. Aesthetic Plast Surg 29:546-551

7. Lemperle G, Morhenn V, Charrier U (2003) Human histology and persistence of various injectable filler substances for soft tissue augmentation. Aesthetic Plast Surg 27:354-366

8. Bains RD, Thorpe H, Southern S (2006) Hand aging: patients' opinions. Plast Reconstr Surg 117:2212-2218

9. Butterwick KJ (2005) Rejuvenation of the aging hand. Dermatol Clin 23:512-527

10. Butterwick KJ (2002) Lipoaugmentation for aging hands: a comparison of the longevity and aesthetic results of centrifuged versus non centrifuged fat. Dermatol Surg 28:987-991

11. Butterwick KJ, Bevin AA, Iyer S (2006) Fat transplantation using fresh versus frozen fat: a side-by-side two-hand comparison pilot study. Dermatol Surg 32:642-644

12. Ellis IR, Schor SL (1996) Differential effects of TGFb1 on hyaluronan synthesis by fetal and adult skin fibroblasts: implications for cell migration and wound healing. Exp Cell Res 228:326-333

13. Chen WY, Abatangelo G (1999) Functions of hyaluronan in wound repair. Wound Repair Regen 7:79-89

14. Williams S, Tamburic S, Stensvik H, Weber M (2009) Changes in skin physiology and clinical appearance after microdroplet placement of hyaluronic acid in aging hands. J Cosmet Dermatol $8: 216-225$

15. Man J, Rao J, Goldman M (2008) A double bind comparative study of nonanimal-stabilized hyaluronic acid versus human collagen for tissue augmentation of the dorsal hands. Dermatol Surg 34:1026-1031

16. Coleman SR (2002) Hand rejuvenation with structural fat grafting. Plast Reconstr Surg 110:1731-1744

17. Adoubib JH, deCastro CC, Gradel J (1992) Hand rejuvenescence by fat filling. Ann Plast Surg 28:559-564

18. Matarasso SL, Carruthers JD, Jewell ML (2006) Restylane Consensus Group. Consensus recommendations for soft-tissue augmentation with nonanimal stabilized hyaluronic acid (Restylane). Plast Reconstr Surg 117:1-43

19. Kerscher M, Bayrhammer J, Reuther T (2008) Rejuvenating influence of a stabilized hyaluronic acid-based gel of nonanimal origin on facial skin aging. Dermatol Surg 34:720-726

20. Yoneda M, Yamagata M, Suzuki S, Kimata K (1988) Hyaluronic acid modulates proliferation of mouse dermal fibroblasts in culture. J Cell Sci 90:265-273

21. Wang F, Garza LA, Kang S et al (2007) In vivo stimulation of de novo collagen production caused by cross-linked hyaluronic acid dermal filler injections in photodamaged human skin. Arch Dermatol 143:155-163

22. Christensen L, Breiting V, Janssen M, Vuust J, Hogdall E (2005) Adverse reactions to injectable soft tissue permanent fillers. Aesthetic Plast Surg 29:34-48

23. Lowe NJ, Maxwell CA, Lowe P, Duick MG, Shah K (2001) Hyaluronic acid skin fillers. Adverse reactions and skin testing. J Am Acad Dermatol 45:930-933

24. Brody HJ (2005) Use of hyaluronidase in the treatment of granulomatous hyaluronic acid reactions. Dermatol Surg 31(8 Pt 1): 893-897

25. Lupton JR, Alster TS (2000) Cutaneous hypersensitivity reaction to injectable hyaluronic acid gel. Dermatol Surg 26:135-137

26. De Boulle K (2004) Management of complications after implantation of fillers. J Cosmet Dermatol 3:2-15 